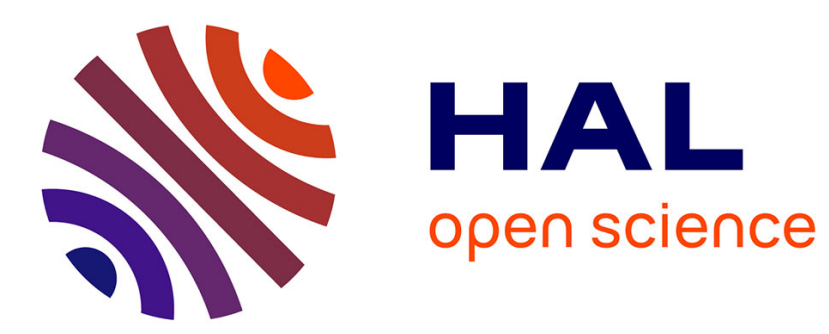

\title{
The phase retrieval problem for the Radar Ambiguity Function and vice versa \\ Philippe Jaming
}

\section{To cite this version:}

Philippe Jaming. The phase retrieval problem for the Radar Ambiguity Function and vice versa. 2010 IEEE International Radar Conference "Global Innovation in Radar", 2010, Washington, France. pp.230 - 235, 10.1109/RADAR.2010.5494619 . hal-01104104

\section{HAL Id: hal-01104104 https://hal.science/hal-01104104}

Submitted on 16 Jan 2015

HAL is a multi-disciplinary open access archive for the deposit and dissemination of scientific research documents, whether they are published or not. The documents may come from teaching and research institutions in France or abroad, or from public or private research centers.
L'archive ouverte pluridisciplinaire HAL, est destinée au dépôt et à la diffusion de documents scientifiques de niveau recherche, publiés ou non, émanant des établissements d'enseignement et de recherche français ou étrangers, des laboratoires publics ou privés. 


\title{
The Phase Retrieval Problem for the Radar Ambiguity Function and vice versa
}

\author{
Philippe JAMING \\ Université dOrléans \\ Laboratoire MAPMO, CNRS, UMR 6628, Fédération Denis Poisson, FR 2964 \\ Bâtiment de Mathématiques, BP 6759 \\ 45067 Orléans cedex 2, FRANCE \\ Email: Philippe.Jaming@univ-orleans.fr \\ http://jaming.nuxit.net
}

\begin{abstract}
The first part of this note is based on a joint paper with A. Bonami and G. Garrigós [3] in which the phase retrieval problem for the Radar Ambiguity Function (i.e. the Radar Ambiguity Problem) has been tackled. In particular it was shown that for wide classes of signals, the radar ambiguity problem has a unique solution, up to trivial transformations.

In the second part of this note, we report on ongoing work by the author where the radar ambiguity function is used as a tool to solve other phase retrieval problems.
\end{abstract}

\section{INTRODUCTION}

In this paper, we report on our work on the phase retrieval problem for the Radar Ambiguity Function and for Fractional Fourier Transforms. Let us recall that the ambiguity function of $u \in L^{2}(\mathbb{R})$ is defined by

$$
A(u)(x, y)=\int_{\mathbb{R}} u\left(t+\frac{x}{2}\right) \overline{u\left(t-\frac{x}{2}\right)} e^{-2 i \pi t y} \mathrm{~d} t .
$$

This function has been introduced by Woodward [12] in Radar theory and has been studied by many authors. The properties that we use here are well documented see e.g. [1], [11]. The first problem under consideration here is the phase retrieval problem for the ambiguity function:

Problem 1: Radar ambiguity Problem.

Given $u$ in some subset $\mathcal{D} \subset L^{2}(\mathbb{R})$, find all $v \in \mathcal{S}$ such that

$$
|A(v)(x, y)|=|A(u)(x, y)| \quad \text { for all } x, y \in \mathbb{R} .
$$

We then say that $v$ is a partner of $u($ in $\mathcal{D})$.

It is not hard to see that $v$ defined by $v(x)=$ $c e^{i b x} u(\varepsilon x-a), \varepsilon= \pm 1, a, b \in \mathbb{R}, c \in \mathbb{C}$ with $|c|=1$ is a partner of $u$, in which case we say that $v$ is a trivial partner of $u$. The question we ask is thus whether nontrivial partners exist and to have as large as possible classes $\mathcal{D}$ of functions which have only trivial partners. The classes we consider are mainly the two following:

i) Hermite functions that is $\mathcal{D}$ is the set of all functions of the form $P(x) e^{-\pi x^{2}}, P$ a polynomial;

ii) rectangular pulse trains, that is $\mathcal{D}$ is the set of all functions of the form $\sum_{k=1}^{N} a_{k} \chi_{(k, k+\eta)}$ where the $a_{k}$ 's are complex numbers and $\chi_{(k, k+\eta)}$ is the characteristic function of the interval $(k, k+\eta)$ where $0<\eta \leq 1 / 2$.

The second family of problems we deal with is the Phase Retreival Problem for the Fractional Fourier Transform (FrFT). This transform is defined as follows: let $\alpha \in \mathbb{R} \backslash \pi \mathbb{Z}$, let $c_{\alpha}=\frac{\exp \frac{i}{2}\left(\alpha-\frac{\pi}{2}\right)}{\sqrt{|\sin \alpha|}}$. For $u \in L^{1}\left(\mathbb{R}^{d}\right)$ and $\alpha \notin \pi \mathbb{Z}$, define

$$
\begin{aligned}
\mathcal{F}_{\alpha} u(\xi)= & c_{\alpha} e^{-i \pi|\xi|^{2} \cot \alpha} \times \\
& \times \int_{\mathbb{R}} u(t) e^{-i \pi t^{2} \cot \alpha} e^{-2 i \pi t \xi / \sin \alpha} \mathrm{d} t .
\end{aligned}
$$

Note that $\mathcal{F}_{\pi / 2}$ is just $\mathcal{F}_{\pi / 2}=\mathcal{F}$, the usual Fourier Transform. As $\left\|\mathcal{F}_{\alpha} u\right\|_{L^{2}(\mathbb{R})}=\|u\|_{L^{2}(\mathbb{R})}$ this transform can be extended to $u \in L^{2}(\mathbb{R})$. The problem we address here is the following:

Problem 2: Phase Retrieval Problem for the fractional Fourier transform.

Let $u, v \in L^{2}(\mathbb{R})$ and let $\tau \subset[0, \pi)$ be a set of indices (finite or not). Assume that $\left|\mathcal{F}_{\alpha} v\right|=\left|\mathcal{F}_{\alpha} u\right|$ for every $\alpha \in \tau$.

i) Does this imply that $v=c u$ for some constant $c \in$ $\mathbb{C},|c|=1$ ?

ii) If we restrict $u \in \mathcal{D}$ for some set $\mathcal{D} \subset L^{2}(\mathbb{R})$ do we then have $v=c u$ for some constant $c \in \mathbb{C},|c|=1$ ?

iii) If we further restrict both $u, v \in \mathcal{D}$ for some set $\mathcal{D} \subset L^{2}(\mathbb{R})$ do we then have $v=c u$ for some constant $c \in \mathbb{C},|c|=1$ ? 
In the first two cases we say that $u$ is uniquely determined (up to constant multiples or up to a constant phase factor) from $\left\{\left|\mathcal{F}_{\alpha} u\right|, \alpha \in \tau\right\}$. In the last case we say that $u$ is uniquely determined (up to a constant phase factor) from $\left\{\left|\mathcal{F}_{\alpha} u\right|, \alpha \in \tau\right\}$ within the class $\mathcal{D}$.

This problem appears in diffraction optics and quantum mechanics. We will reformulate this problem in terms of the radar ambiguity function. This will allow us to bring a solution to the problem for various classes $\mathcal{D}$ of functions. We will again focus on the class of Hermite functions and of rectangular pulse trains and also give some results for more general compactly supported functions.

The remaining of this paper is organized as follows: the next section is devoted to the radar ambiguity functions and presents results that already appeared in [3]. Section 3 is then devoted to the announcement of new results from [10].

\section{THE RADAR AMBIGUITY PROBLEM}

The radar ambiguity problem as stated is not yet fully solved. Early results can be found in [6], [4]. The author tackled this problem in [9], [7] and [3]. The aim of this section is to present the two main results from this last paper which may so far have stayed unnoticed outside the mathematical community and all results presented in this section come from that paper. The main object of [3] was to study the Radar Ambiguity Problem in a "discrete" setting by restricting attention to Hermite functions and to pulse trains.

\section{A. Hermite functions}

In this section, we study Problem 1 when $u$ is of the form $u(x)=P(x) e^{-\pi x^{2}}$ where $P$ is a polynomial. As a consequence of Hardy's Uncertainty Principle for the ambiguity function (see e.g. [2], [8]), it is not hard to see that every partner $v$ is of the form $v(x)=$ $Q(x) e^{(a+i b) x-\pi x^{2}}$ with $Q$ a polynomial. Thus, up to replacing $v$ by a trivial partner, we may assume that $v(x)=Q(x) e^{-\pi x^{2}}$. Moreover, it is easy to see that $P$ and $Q$ have same degree.

If we write $H_{j}=(-1)^{j} e^{\pi t^{2}} \partial_{t}^{j} e^{-2 \pi t^{2}}$ for the Hermite basis, then we may write $u$ and $v$ in that basis, $u=$ $\sum_{j=0}^{n} \alpha_{j} H_{j}$ and $v=\sum_{j=0}^{n} \beta_{j} H_{j}$. Define $\mathcal{P}=\sum_{j=0}^{n} \alpha_{j} t^{j}, \mathcal{Q}=$ $\sum_{j=0}^{n} \beta_{j} t^{j}$, and let us write $x, y \in \mathbb{R}, Z=x+i y, \bar{Z}=$ $x-i y$. One may then show that

$$
A(u)(x, y)=\sum_{j=0}^{n} \frac{2^{-j}}{j !} \mathcal{P}^{(j)}(Z) \overline{\mathcal{P}^{(j)}(-Z)} e^{|Z|^{2} / 4} .
$$

Next, expending $|A(u)|^{2}$, the radar ambiguity problem then amounts to determining $\beta$ such that

$$
\begin{aligned}
& \sum \frac{2^{-j-k}}{j ! k !} \mathcal{P}^{(k)}(-Z) \mathcal{P}^{(j)}(Z) \overline{\mathcal{P}^{(j)}(-Z) \mathcal{P}^{(k)}(Z)} \\
= & \sum \frac{2^{-j-k}}{j ! k !} \mathcal{Q}^{(k)}(-Z) \mathcal{Q}^{(j)}(Z) \overline{\mathcal{Q}^{(j)}(-Z) \mathcal{Q}^{(k)}(Z)} .
\end{aligned}
$$

Expending this polynomial in $Z$ and $\bar{Z}$ and comparing highest order terms leads to $\left|\beta_{n}\right|=\left|\alpha_{n}\right|$. Up to replacing $v$ by a trivial partner, we may thus assume that $\beta_{n}=\alpha_{n}$.

Expending (1) further, we then obtain

$$
\mathcal{P}(Z) \mathcal{P}(-Z)=\mathcal{Q}(Z) \mathcal{Q}(-Z)
$$

so that the zeroes of $\mathcal{Q}$ are obtained from those of $\mathcal{P}$ via a symmetry, so that we may factor $\mathcal{P}(Z)=$ $A(Z) B(Z) C(Z)$ and $\mathcal{Q}(Z)=A(Z) B(-Z) C(Z)$ where $C(Z)$ is of the form $Z^{k} \prod\left(Z^{2}-\lambda_{j}^{2}\right)$. Our aim is to show that neither $A$ nor $B$ are even or odd.

For a polynomial $P$, write $\tilde{P}(Z)=P(Z) P^{\prime}(-Z)+$ $P(-Z) P^{\prime}(Z)$. One easily sees that $\tilde{P}=0$ if and only if $P$ has a definite parity.

Expending further (1), we now obtain

$$
\begin{aligned}
& \mathcal{P}^{\prime}(Z) \mathcal{P}^{\prime}(-Z)+\frac{2}{n} \overline{\alpha_{n-1}} \tilde{\mathcal{P}}(Z) \\
= & \mathcal{Q}^{\prime}(Z) \mathcal{Q}^{\prime}(-Z)+\frac{2}{n} \overline{\beta_{n-1}} \tilde{\mathcal{Q}}(Z) .
\end{aligned}
$$

We then reformulate this in terms of $A$ and $B$ and notice that the equations obtained that way generically do not have a solution. This leads to the following result:

Theorem 2.1: [3] For almost all and quasi-all polynomials $P, u(t)=P(t) e^{-\pi t^{2}}$ has only trivial partners

Here, for a fixed degree $n$, almost-all refers to 0 Lebesgue measure in $\mathbb{C}^{n+1}$, while quasi-all refers to Baire theory. Actually we showed that the set of polynomials for which there exists a non trivial partner is included in a lower dimensional sub-manifold of $\mathbb{C}^{n+1}$. We conjecture that this manifold is actually empty so that the above result is true for all polynomials.

There are some polynomials $P$ for which one can assert that $u(t)=P(t) e^{-\pi t^{2}}$ has only trivial partners, for instance those that have no term of degree $n-1$. From this, it is not hard to deduce the following:

Corollary 2.2: [3] The set of functions in $L^{2}(\mathbb{R})$ that has only trivial partners is dense in $L^{2}(\mathbb{R})$. 


\section{B. Pulse trains}

In this section, we consider signals of the form $u(t)=$ $\sum a_{j} H(t-j)$ where $H$ has support in $[0,1 / 2]$. One then easily shows that, for $y \in \mathbb{R}, k \in \mathbb{Z}$ and $k-\frac{1}{2} \leq x \leq$ $k+\frac{1}{2}$,

$$
A(u)(x, y)=\left(\sum_{j \in \mathbb{Z}} a_{j} \overline{a_{j-k}} e^{2 i \pi j y}\right) A(H)(x-k, y)
$$

This lead us to propose the following Discrete Radar Ambiguity Problem in [7]:

Problem 3: Discrete Radar Ambiguity Problem. Given $a=\left\{a_{j}\right\} \in \ell^{2}(\mathbb{Z})$, find all sequences $b \in \ell^{2}(\mathbb{Z})$ such that, for every $k \in \mathbb{Z}$ and $y \in \mathbb{R}$,

$$
|\mathcal{A}(a)(k, y)|=|\mathcal{A}(b)(k, y)|
$$

where $\mathcal{A}(a)(k, y)=\sum_{j \in \mathbb{Z}} a_{j} \overline{a_{j-k}} e^{2 i \pi j y}$.

We will then say that $a$ and $b$ are partners and that they are trivial partners if $b_{j}=e^{i \beta+i j \omega} a_{ \pm j-\ell}$ for some $\beta, \omega \in \mathbb{R}$ and $\ell \in \mathbb{Z}$ and a choice of \pm .

One easily sees that, as $\mathcal{A}(a)$ and $\mathcal{A}(b)$ have same support, if the support of $a$ has finite length $N$ then so has $b$. More precisely, we will say that $a \in \mathcal{S}(N)$ if $a$ has support $\{0, \ldots, N\}$ with $a_{0} a_{N} \neq 0$. Then, if $b$ is a partner of $a$, up to replacing it by a trivial partner, we may also assume that $b \in \mathcal{S}(N)$.

By adapting a method by Bueckner [4] from the continuous case, the discrete radar ambiguity problem can be reformulated as a combinatorial problem on matrices. More precisely, let

$$
d_{j, k}=\left\{\begin{array}{ll}
a_{\frac{j+k}{2}} a_{\frac{j-k}{2}} & \text { if } j, k \text { have same parity } \\
0 & \text { otherwise }
\end{array},\right.
$$

and let $K_{a}=\left[d_{j, k}\right]_{-N \leq j, k \leq N}$ and call this the Ambiguity Matrix of $a$. One then shows that

Proposition 2.3: [3], [7] Two sequences $a, b \in \mathcal{S}(N)$ are partners if and only if $K_{a}^{*} K_{a}=K_{b}^{*} K_{b}$. In other words, if $V_{i}\left(\right.$ resp. $\left.W_{i}\right)$ is the $i$-th column of $K_{a}$ (resp. $K_{b}$ ) this is equivalent to the following identity for all $(i, j)$ 's:

$$
(i, j) \quad\left\langle V_{i}, V_{j}\right\rangle=\left\langle W_{i}, W_{j}\right\rangle .
$$

Recall that the Kronecker product of two matrices $A=$ $\left[a_{i, j}\right]_{-N \leq i, j \leq N}$ and $B$ is the matrix defined blockwise by

$$
A \otimes B=\left[\begin{array}{cccc}
a_{1,1} B & a_{1,2} B & \ldots & a_{1, n} B \\
a_{2,1} B & a_{2,2} B & \ldots & a_{2, n} B \\
\vdots & \vdots & \ddots & \vdots \\
a_{n, 1} B & a_{n, 2} B & \ldots & a_{n, n} B
\end{array}\right] .
$$

The main result of [3], [7] for pulse type signals can be stated as follows:
Theorem 2.4: [3], [7] Let $a \in \mathcal{S}(N)$ and $b \in \mathcal{S}(M)$ ( $N, M$ integers) then there is a $c \in \mathcal{S}(M(2 N+1)+N)$ such that $K_{a} \otimes K_{b}=K_{c}$. Let us denote $c=a \otimes b$. Then, if $a, a^{\prime}$ are partners and $b, b^{\prime}$ are partners, then $a \otimes b$ and $a^{\prime} \otimes b^{\prime}$ are partners. Moreover, even if $a, a^{\prime}$ (resp. $b, b^{\prime}$ ) are trivial partners, $a \otimes b$ and $a^{\prime} \otimes b^{\prime}$ need not be trivial partners.

Proof Let us just prove that $a \otimes b$ and $a^{\prime} \otimes b^{\prime}$ are partners if $a, a^{\prime}$ (resp. $b, b^{\prime}$ ) are partners. This follows from standard properties of the Kronecker product:

$$
\begin{aligned}
K_{a^{\prime} \otimes b^{\prime}}^{*} K_{a^{\prime} \otimes b^{\prime}} & =\left(K_{a^{\prime}} \otimes K_{b^{\prime}}\right)^{*}\left(K_{a^{\prime}} \otimes K_{b^{\prime}}\right) \\
& =\left(K_{a^{\prime}}^{*} \otimes K_{b^{\prime}}^{*}\right)\left(K_{a^{\prime}} \otimes K_{b^{\prime}}\right) \\
& =\left(K_{a^{\prime}}^{*} K_{a^{\prime}}\right) \otimes\left(K_{b^{\prime}}^{*} K_{b^{\prime}}\right) \\
& =\left(K_{a}^{*} K_{a}\right) \otimes\left(K_{b}^{*} K_{b}\right) .
\end{aligned}
$$

Unwinding the computation, we immediately get that $K_{a^{\prime} \otimes b^{\prime}}^{*} K_{a^{\prime} \otimes b^{\prime}}=K_{a \otimes b}^{*} K_{a \otimes b}$.

Actually $c=a \otimes b$ may be constructed as follows. Write $P(z)=\sum_{k=0}^{N} a_{k} z^{k}$ and $Q(z)=\sum_{k=0}^{M} b_{k} z^{k}$. Then $P(z) Q\left(z^{2 N+1}\right)=\sum_{k=0}^{M(2 N+1)+N} c_{k} z^{k}$. For example, if $a=(1,2), b=(1,2)$ and $a^{\prime}=(2,1)$ (a trivial partner of $a$ ) then $a \otimes b=(1,2,0,2,4)$ while $a^{\prime} \otimes b=(2,4,0,1,2)$ and these two sequences are not trivial partners.

Further, based on this construction, we are able to prove the following which has to be compared to Corollary 2.2:

Corollary 2.5: [3] The set of functions in $L^{2}(\mathbb{R})$ that has non-trivial partners is dense in $L^{2}(\mathbb{R})$.

Finally, we were also able to obtain a result for the ambiguity problem itself, without assuming that $v$ is itself of pulse type. More precisely:

Theorem 2.6: [3] Let $0<\eta \leq \frac{1}{3}$ and $u(t)=$ $\sum_{j=0}^{N} a_{j} \chi_{[j, j+\eta]}(t)$ where $a=\left(a_{0}, a_{1}, \ldots, a_{N}\right) \in \mathbb{C}^{N+1}$. Let $v \in L^{2}(\mathbb{R})$ be a trivial partner of $u$. Then, up to replacing $v$ by a trivial partner, $v$ is of the form $v=$ $\sum_{j=0}^{N} b_{j} \chi_{[j, j+\eta]}$, where $b=\left(b_{0}, b_{1}, \ldots, b_{N}\right) \in \mathbb{C}^{N+1}$ is a partner of $a$.

\section{THE RADAR AMBIGUITY FUNCTION AND THE PHASE RETRIEVAL PROBLEM FOR FRACTIONAL FOURIER TRANSFORMS}

The aim of this section is to report on ongoing work [10] on the phase retrieval for the Fractional Fourier Transform (FrFT). Note that, mathematically, there is no difference between the phase retrieval problem for the Fractional Fourier Transform and the same problem for the classical Fourier Transform. We here concentrate on 
the question whether several phase-less measurements can lead to uniqueness results.

The link between the FrFT and the ambiguity function comes from the following property:

$$
\begin{aligned}
& A\left(\mathcal{F}_{\alpha} u, \mathcal{F}_{\alpha} v\right)(x, y) \\
& \quad=A(u, v)(x \cos \alpha-y \sin \alpha, x \sin \alpha+y \cos \alpha) .
\end{aligned}
$$

In particular,

$$
\begin{aligned}
A(u)(-y \sin \alpha, y \cos \alpha) & =A\left(\mathcal{F}_{\alpha} u\right)(0, y) \\
& =\mathcal{F}\left[\left|\mathcal{F}_{\alpha} u\right|^{2}\right](y) .
\end{aligned}
$$

It follows that Problem 2 amounts to being able to reconstruct $u$ from the knowledge of its ambiguity function on certain lines going through the origin.

One of the main results from [10] is the following:

Theorem 3.1: In the following cases, exact reconstruction can be obtained.

1) Let $u, v \in L^{2}(\mathbb{R})$ such that, for every $\alpha \in$ $[-\pi / 2, \pi / 2],\left|\mathcal{F}_{\alpha} v\right|=\left|\mathcal{F}_{\alpha} u\right|$. Then there exists $c \in \mathbb{C}$ with $|c|=1$ such that $v=c u$.

2) Let $\tau \subset[-\pi / 2, \pi / 2]$ be either a set of positive measure or a set with an accumulation point $\alpha_{0} \neq 0$. Let $u, v \in L^{2}(\mathbb{R})$ with compact support such that, for every $\alpha \in \tau,\left|\mathcal{F}_{\alpha} v\right|=\left|\mathcal{F}_{\alpha} u\right|$. Then there exists $c \in \mathbb{C}$ with $|c|=1$ such that $v=c u$.

3) Let $a>0$ and define $\left(\alpha_{k}\right)_{k \in \mathbb{Z}}$ by $\alpha_{0}=\pi / 2$ and, for $k \in \mathbb{Z} \backslash\{0\}, \alpha_{k}=\arctan \frac{a^{2}}{k}$. Then, given $u, v \in$ $L^{2}(\mathbb{R})$ with compact support included in $[-a, a]$, if $\left|\mathcal{F}_{\alpha_{k}} v\right|=\left|\mathcal{F}_{\alpha_{k}} u\right|$ for every $k \in \mathbb{Z}$, then there exists $c \in \mathbb{C}$ with $|c|=1$ such that $v=c u$.

Proof From (3), if $\left|\mathcal{F}_{\alpha} v\right|=\left|\mathcal{F}_{\alpha} u\right|$ for every $\alpha \in$ $[-\pi / 2, \pi / 2]$ then, from (3), we get $A(v)=A(u)$. This, as is well known, implies that $v=c u$ for some complex number with $|c|=1$.

Let us prove the third part of the Theorem (the second part follows from a similar argument). Note that $\varphi_{x}(t):=u\left(t+\frac{x}{2}\right) \overline{u\left(t-\frac{x}{2}\right)}$ is an $L^{1}$ function supported in the interval $\left[-a+\frac{|x|}{2}, a-\frac{|x|}{2}\right]$ when $|x|<2 a$ and is 0 for $|x| \geq 2 a$. But then, from the Shannon-Whittaker Formula, the Fourier transform $A(u)(x, y)$ of $\varphi_{x}$ may be reconstructed from its samples. More precisely,

$$
\begin{aligned}
& A(u)(x, y)=\widehat{\varphi_{x}}(y) \\
= & \sum_{k \in \mathbb{Z}} \widehat{\varphi_{x}}\left(\frac{h_{x} k}{2}\right) \operatorname{sinc} \frac{2 \pi}{h_{x}}\left(y+\frac{h_{x} k}{2}\right) \\
= & \sum_{k \in \mathbb{Z}} A(u)\left(x, \frac{h_{x} k}{2}\right) \operatorname{sinc} \frac{2 \pi}{h_{x}}\left(y+\frac{h_{x} k}{2}\right)
\end{aligned}
$$

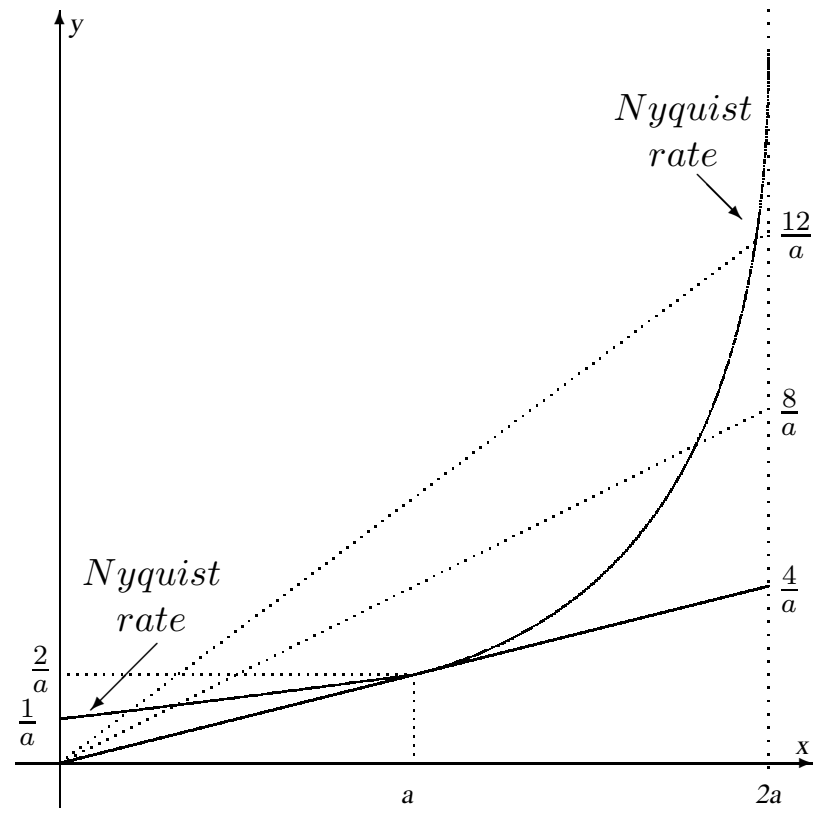

Fig. 1. The Nyquist rate and the sampling lines for $A(u)$

provided $\left|h_{x}\right| \leq \frac{1}{a-|x| / 2}$. A similar expression holds for $A(v)(x, y)$.

Now recall from (3) that

$$
\begin{aligned}
\mathcal{F}\left[\left|\mathcal{F}_{-\alpha} u\right|^{2}\right](\xi) & =A\left(\mathcal{F}_{-\alpha} u\right)(0, \xi) \\
& =A(u)(\xi \sin \alpha, \xi \cos \alpha) .
\end{aligned}
$$

In particular, if we chose $h_{x}$ of the form $h_{x}=\gamma x$ then,

$$
\begin{aligned}
A(u)\left(x, \frac{h_{x} k}{2}\right) & =A(u)\left(x, \frac{\gamma k}{2} x\right) \\
& =\mathcal{F}\left[\left|\mathcal{F}_{-\alpha_{k}} u\right|^{2}\right]\left(x / \sin \alpha_{k}\right)
\end{aligned}
$$

where $\alpha_{k}=\arctan \frac{2}{k \gamma}\left(\alpha_{0}=-\pi / 2\right)$.

As we assumed that $\left|\mathcal{F}_{-\alpha_{k}} v\right|=\left|\mathcal{F}_{-\alpha_{k}} u\right|$ for every $k$, it follows that $A(v)\left(x, \frac{h_{x} k}{2}\right)=A(u)\left(x, \frac{h_{x} k}{2}\right)$ for every $x$ and $k$. From (4) we then deduce that $A(v)=A(u)$ everywhere thus $v=c u$ with $c \in \mathbb{C},|c|=1$.

It remains to choose $\gamma$ so that $|\gamma x| \leq \frac{1}{a-|x| / 2}$ for $0 \leq x \leq 2 a$. For this, it is enough to find the point on the hyperbola $y=1 /(a-x / 2)$ at which the tangent goes through the origin. Easy calculus shows that this point is $x=a, y=2 / a$, thus any $\gamma \leq \frac{2}{a^{2}}$ will do (see Figure 1).

Note that in the course of the proof of the last point, we have established a reconstruction formula. However, to obtain a reasonably accurate reconstruction, we need 
the number of measures $\left|\mathcal{F}_{\alpha_{k}} u\right|$ to be rather large, making this reconstruction formula non practicable.

If the signal is assumed to be more "structured", then one or two well chosen measures actually suffice. In the following theorem we group the main results from [10] in that direction.

Theorem 3.2: In the following cases, exact reconstruction can be obtained.

1) Assume $u, v$ are Hermite functions and let $\alpha \in \mathbb{R} \backslash$ $\mathbb{Q} \pi$. If $|v|=|u|$ and $\left|\mathcal{F}_{\alpha} v\right|=\left|\mathcal{F}_{\alpha} u\right|$ then $v=c u$ with $|c|=1$.

2) Assume $u, v$ are rectangular pulse trains $\sum c_{j} \chi_{[j, j+\eta)}$ with $0<\eta \leq \frac{1}{2}$ and let $\alpha \in \mathbb{R} \backslash \frac{\pi}{2} \mathbb{Z}$. If $\left|\mathcal{F}_{\alpha} v\right|=\left|\mathcal{F}_{\alpha} u\right|$ then $v=c u$ with $|c|=1$.

3) Assume $u, v$ are of the form $\sum_{\text {finite }} c_{i} e^{-\pi\left(t-t_{i}\right)^{2}}$, $c_{i} \in \mathbb{C}$ and $t_{i} \in \mathbb{R}$, and let $\alpha \in \mathbb{R} \backslash \frac{\pi}{2} \mathbb{Z}$. If $\left|\mathcal{F}_{\alpha} v\right|=$ $\left|\mathcal{F}_{\alpha} u\right|$ then $v=c u$ with $|c|=1$.

The assumptions on $\alpha$ in this theorem are sharp. For instance, Pauli asked whether $|v|=|u|$ and $|\hat{v}|=|\hat{u}|$ implies $v=c u, c \in \mathbb{C}$ with $|c|=1$. It turns out that there are counterexamples to this where both $u$ and $v$ are of the form $\sum c_{i} e^{-\pi\left(t-t_{i}\right)}$ as well as in the case where both $u$ and $v$ are pulse trains (see e.g. [9] and [5] for the state of the art).

Let us now sketch a proof of this theorem.

Proof Assume first that both $u$ and $v$ are Hermite functions and expend them in the Hermite basis: $u(t)=$ $\sum_{j=0}^{N} c_{j} H_{j}$ and $v(t)=\sum_{j=0}^{M} c_{j} H_{j}$. Then, as is well known,

$$
\mathcal{F}_{\theta}[u](t)=\sum_{k=0}^{N} c_{k} e^{-i k \theta} H_{k}(t)
$$

and a similar expression holds for $\mathcal{F}_{\theta}[v]$. Then $|v|^{2}=$ $|u|^{2}$ and $\left|\mathcal{F}_{\theta}[v]\right|^{2}=\left|\mathcal{F}_{\theta}[u]\right|^{2}$ is equivalent to

$$
\left\{\begin{array}{c}
\sum_{j, k=1}^{M} d_{j} \overline{d_{k}} H_{j}(t) H_{k}(t) \\
=\sum_{j, k=1}^{N} c_{j} \overline{c_{k}} H_{j}(t) H_{k}(t) \\
\sum_{j, k=1}^{M} d_{j} \overline{d_{k}} e^{i(k-j) \beta} H_{j}(t) H_{k}(t) \\
=\sum_{j, k=1}^{N} c_{j} \overline{c_{k}} e^{i(k-j) \beta} H_{j}(t) H_{k}(t)
\end{array}\right.
$$

Looking at the highest order term (of the polynomial part) in (5) we obtain $\left|d_{M}\right|^{2} H_{M}^{2}(t)$ on the left hand side and $\left|c_{N}\right|^{2} H_{N}(t)^{2}$ so that $M=N$ and $\left|d_{M}\right|=\left|c_{N}\right|$.
Up to replacing $v$ by $\frac{c_{N}}{d_{N}} v$ we may thus assume that $d_{N}=c_{N}$.

The proof then proceeds by a down-going induction. If $d_{N-j}=c_{N-j}$ for $j=0, \ldots, k-1$, then we may simplify all terms in (5) which only depend on those coefficients. The highest order term that is left is $H_{N-k} H_{N}$ and comparing this term reduces (5) to

$$
\left\{\begin{aligned}
\operatorname{Re}\left(d_{N} \overline{d_{N-k}}\right) & =\operatorname{Re}\left(c_{N} \overline{c_{N-k}}\right) \\
\operatorname{Re}\left(e^{i k \theta} d_{N} \overline{d_{N-k}}\right) & =\operatorname{Re}\left(e^{i k \theta} c_{N} \overline{c_{N-k}}\right)
\end{aligned}\right.
$$

By assumption, $e^{i k \theta} \notin \mathbb{R}$ so that $d_{N} \overline{d_{N-k}}=c_{N} \overline{c_{N-k}}$ thus $d_{N-k}=c_{N-k}$.

Let us now assume that $u$ and $v$ are pulse trains $u(t)=$ $\sum a_{j} H(t-j)$ and $v(t)=\sum b_{j} H(t-j)$ where $a_{j}, b_{j}$ are finite sequences. Recall that, for $x \in\left[j-\frac{1}{2}, j+\frac{1}{2}\right]$, $A(u)(x, y)=e^{i \pi j y} \mathcal{A}(a)(j, y) A\left(\chi_{[0, \eta)}\right)(x-j, y)$ and a similar expression holds for $A(v)$. According to (3), the hypothesis of the theorem translates into

$$
\begin{aligned}
& \mathcal{A}(a)(j, y \cos \alpha) A\left(\chi_{[0, \eta)}\right)(-y \sin \alpha-j, y \cos \alpha) \\
& \mathcal{A}(b)(j, y \cos \alpha) A\left(\chi_{[0, \eta)}\right)(-y \sin \alpha-j, y \cos \alpha)
\end{aligned}
$$

for all $j \in \mathbb{Z}$ and for all $y$ such that $-y \sin \alpha \in\left[j-\frac{1}{2}, j+\frac{1}{2}\right] \quad$ i.e. for all $y \in I_{\alpha, j}:=\left[\frac{-2 j+1}{2 \sin \alpha}, \frac{-2 j-1}{2 \sin \alpha}\right]$. Recall that we assumed that $\alpha$ is not a multiple of $\pi$ so that this is perfectly defined.

On the other hand, for $|x| \leq \eta A\left(\chi_{[0, \eta)}\right)(x, y)=$ $\frac{e^{i \pi \eta y}}{\pi y} \sin \pi(\eta-|x|)$, thus $A\left(\chi_{[0, \eta)}\right)(-y \sin \alpha-j, y \cos \alpha)$ is non zero on a subset $J_{\alpha, j} \subset I_{\alpha, j}$ of positive measure. Thus (6) reduces to

$$
\sum_{k \in \mathbb{Z}} b_{k} \overline{b_{k-j}} e^{2 i \pi k y \cos \alpha}=\sum_{k \in \mathbb{Z}} a_{k} \overline{a_{k-j}} e^{2 i \pi k y \cos \alpha} .
$$

on $J_{\alpha, j}$. As we assumed that $\alpha$ is not a multiple of $\pi / 2$, $\cos \alpha \neq 0$, and as both sides of (7) are trigonometric polynomials, this identity stays true everywhere. As this is the case for every $j, \mathcal{A}(a)=\mathcal{A}(b)$ which is easily seen to imply that $b=c a$ with $c \in \mathbb{C},|c|=1$ thus $v=c u$.

Let us assume that $u(t)=\sum_{j=1}^{N} c_{j} \gamma\left(t-t_{j}\right)$ and $v(t)=\sum_{j=1}^{M} \kappa_{j} \gamma\left(t-\tau_{j}\right)$ with $c_{j}, \kappa_{j} \in \mathbb{C}$ and $t_{j}, \tau_{j} \in \mathbb{R}$. Moreover the $t_{j}$ 's (resp. the $\tau_{j}$ 's) are all distinct. A 
simple computation shows that

$$
\begin{gathered}
A(u)(x, y)=2^{-1 / 2} \sum_{j, k=1}^{N} c_{j} \overline{c_{k}} e^{-\pi\left(t_{j}+t_{k}\right)^{2} / 2} \\
\times e^{-\pi\left(\left(x+t_{k}-t_{j}\right)^{2}+\left(y-i t_{k}-i t_{j}\right)^{2}\right) / 2} .
\end{gathered}
$$

In particular,

$$
\begin{gathered}
A(u)(-t \sin \alpha, t \cos \alpha) \\
=2^{-1 / 2} \sum_{j, k=1}^{N} c_{j} \overline{c_{k}} e^{-\pi\left(t_{j}+t_{k}\right)^{2} / 2} \times \\
\times e^{-\pi\left(\left(-t \sin \alpha+t_{k}-t_{j}\right)^{2}+\left(t \cos \alpha+i t_{k}+i t_{j}\right)^{2}\right) / 2} \\
=\frac{e^{-\pi t^{2} / 2}}{\sqrt{2}} \sum_{j, k=1}^{N} c_{j} \overline{c_{k}} e^{-\pi\left(t_{j}-t_{k}\right)^{2} / 2-i \pi t\left(t_{j} e^{i \alpha}+t_{k} e^{-i \alpha}\right)} .(8)
\end{gathered}
$$

A similar expression holds for $v$ :

$$
\begin{aligned}
& A(v)(-t \sin \alpha, t \cos \alpha)=2^{-1 / 2} e^{-\pi t^{2} / 2} \times \\
& \times \sum_{j, k=1}^{M} \kappa_{j} \overline{\kappa_{k}} e^{-\pi\left(\tau_{j}-\tau_{k}\right)^{2} / 2} e^{-i \pi t\left(\tau_{j} e^{i \alpha}+\tau_{k} e^{-i \alpha}\right)} .
\end{aligned}
$$

The proof is based on two facts.

Fact 1. Let $\alpha \in \mathbb{R} \backslash \frac{\pi}{2} \mathbb{Z}$ and let $\left\{t_{j}\right\}_{j \in \mathbb{Z}}$ and $\left\{\tau_{j}\right\}_{j \in \mathbb{Z}}$ be two finite sequences of real numbers. If there exists $j, k, j^{\prime}, k^{\prime} \in \mathbb{Z}$ such that $t_{j} e^{i \alpha}+t_{k} e^{-i \alpha}=\tau_{j^{\prime}} e^{i \alpha}+\tau_{k^{\prime}} e^{-i \alpha}$ then $t_{j}=\tau_{j^{\prime}}$ and $t_{k}=\tau_{k^{\prime}}$.

This fact is easy to prove. The following fact is well known:

Fact 2. The set $\left\{e^{z t}\right\}_{z \in \mathbb{C}}$ is linearly independent set of functions on $\mathbb{R}$.

From Fact 1, each term of the form $e^{z t}$ appearing in the sum (8) -resp. (9)- appears exactly ones. Moreover, as $\left|\mathcal{F}_{\alpha} v\right|=\left|\mathcal{F}_{\alpha} u\right|$ implies $A(v)(-t \sin \alpha, t \cos \alpha)=$ $A(u)(-t \sin \alpha, t \cos \alpha)$, Fact 2 implies that the two sums are equal term by term: $M=N,\left\{\tau_{j}\right\}=\left\{t_{j}\right\}$ thus (up to renumbering) $\tau_{j}=t_{j}$ and then, for every $j, k=$ $1, \ldots, N, \kappa_{j} \overline{\kappa_{k}}=c_{j} \overline{c_{k}}$. This last identity implies that there exists $c \in \mathbb{C}$ with $|c|=1$ such that $\kappa_{j}=c c_{j}$ for all $j$ and finally that $v=c u$.

Note that so far we have no reasonable algorithm to obtain $u$ from the measures, an issue we plan to tackle in the near future.

\section{CONCLUSiON}

In this paper we have partially solved the phase retrieval problem for the radar ambiguity function. More precisely, we concentrated our attention on the two common cases (gaussian signals and rectangular pulse trains).
In both cases, we have proved that most signals have only trivial partners, if one restricts the problem to these classes of functions. In the case of pulse type signals, we have both the rareness of functions with strange partners, some criteria to have only trivial solutions and various ways to construct functions that have strange partners. Moreover, if the pulses are short enough, then the signal can be reconstructed among all signals (not just pulse trains).

In the second part of the paper, we have used the radar ambiguity function to reconstruct a signal from the modulus of its Fractional Fourier Transforms of various orders. We have shown that, if the orders are well chosen, then uniqueness occurs in several classes of functions, including gaussian signals and pulse trains.

\section{ACKNOWLEDGMENT}

The author was partially supported by the French ANR project AHPI (Analyse Harmonique pour les Problems Inverses).

\section{REFERENCES}

[1] L. Auslander \& R. TOlimieri Radar ambiguity functions and group theory. SIAM J. Math Anal, 16 (1985), 577-601.

[2] A. Bonami, B. Demange \& Ph. Jaming Hermite functions and uncertainty principles for the Fourier and the windowed Fourier transforms. Revista Mat. Iberoamericana, 19 (2003), 23-55.

[3] A. Bonami, G. Garrigós \& Ph. Jaming Discrete radar ambiguity problems. Appl. Comput. Harmon. Anal. 23 (2007), 388-414.

[4] H. F. BUECKNER Signals having the same ambiguity functions. Technical Report 67-C-456, General Electric, Research and Development Center, Schnectady, N.Y., 1967.

[5] J. V. CORBETT The Pauli problem, state reconstruction and quantum-real numbers. Rep. Math. Phys., 57 (2006), 53-68.

[6] R. DE BUDA Signals that can be calculated from their ambiguity function. IEEE Trans. Information Theory, IT16 (1970), 195202.

[7] G. Garrigós, Ph. Jaming \& J.-B. Poly Zéros de fonctions holomorphes et contre-exemples en théorie des radars. In Actes des rencontres d'analyse complexe, Atlantique, Poitiers, 81-104, 2000. Available on

http://hal.ccsd.cnrs.fr/ccsd-00007482

[8] Gröchenig, K. AND Zimmermann, G. Hardy's theorem and the short-time Fourier transform of Schwartz functions. J. London Math. Soc. (2) 63 (2001), 93-103.

[9] PH. JAMING Phase retrieval techniques for radar ambiguity functions. J. Fourier Anal. Appl., 5 (1999), 313-333.

[10] PH. JAming Exact reconstruction from few phase-less measurements. In preparation.

[11] C. H. WILCOX The synthesis problem for radar ambiguity functions. MRC Tech. Summary Report 157 (1960), republished in Radar and Sonar part I (eds. R. Blahut, W. Miller and C. Wilcox), I.M.A. vol in Math. and its Appl. 32, 229-260, Springer, New York, 1991. 
[12] P. M. WOODWARD Probability and Information Theory with Applications to RADAR. Pergamon, 1953. 